\title{
Surgical Approach to Cervical Esophagogastric Anastomoses for Post-esophagectomy Complications
}

\author{
Yukinori Yamagata • Yoshiyuki Kawashima • \\ Toshimasa Yatsuoka Yoji Nishimura • \\ Katsumi Amikura • Hirohiko Sakamoto • \\ Yoichi Tanaka • Yasuyuki Seto
}

Received: 4 November 2012 / Accepted: 17 February 2013 / Published online: 5 March 2013

(C) 2013 The Author(s). This article is published with open access at Springerlink.com

\begin{abstract}
In gastric tube reconstruction, anastomotic leakage and stricture occasionally occur. Additionally, new or recurrent cancer may occur in the esophageal remnant or at the anastomotic site. Such complications, after cervical anastomoses, led to our adoption of a procedure to approach the anastomosis by manubrium and proximal left clavicle resection. This procedure was applied to seven patients between April 2000 and March 2011. The mean age of the patients was 69.9 years (range, 6576 years); all were men. The mean operative time was $506 \mathrm{~min}$ (range, 374-845 min), with an average blood loss of $297 \mathrm{ml}$ (range, 180-606 ml). Esophagogastric anastomoses were performed in two cases, and free jejunal graft transplantations were performed in the remaining five cases; oral intake became possible for all patients. Limited range of motion or other movement disorders of the neck and upper limbs, due to the upper sternum and clavicle resection, were not observed. This invasive surgical procedure can be acceptable for patients who are facing life-threatening consequences or significant decreases in quality of life and are resistant to conservative treatment.
\end{abstract}

Keywords Cervical esophagogastric anastomosis · Manubrium resection $\cdot$ Anastomotic stricture $\cdot$ Remnant esophageal cancer

\section{Introduction}

In general, the gastric tube is used for reconstruction after an esophagectomy, and the reconstruction may be performed via the posterior mediastinum, retrosternal, or presternal route. As the posterior mediastinum route is the shortest and most natural, this route is most often chosen. However,

Y. Yamagata $\cdot$ Y. Kawashima $\cdot$ T. Yatsuoka $\cdot$ Y. Nishimura $\cdot$ $\mathrm{K}$. Amikura $\cdot \mathrm{H}$. Sakamoto $\cdot$ Y. Tanaka

Division of Gastroenterological Surgery, Saitama Cancer Center, Saitama, Japan

Y. Yamagata $(\bowtie) \cdot$ Y. Seto

Department of Gastrointestinal Surgery, Graduate School

of Medicine, The University of Tokyo, 7-3-1 Hongo, Bunkyo-ku,

Tokyo 113-8655, Japan

e-mail: yamagay-tky@umin.ac.jp in cases of locally advanced esophageal cancer where there are concerns about local recurrence, the retrosternal route may be chosen in order to minimize the risk of tumor invasion or irradiation of the gastric tube. In which route, we adopted a cervical anastomosis, so that the anastomosis was located behind the sternum.

In gastric tube reconstructions, slow peripheral circulation of the gastric tube, tension, and anastomotic complications, such as leakage or stricture, occasionally occur. Leakage induces local inflammation around the anastomosis, with scarring occurring as a result of the inflammation; ultimately an anastomotic stricture is formed. The bend in the anastomosis occasionally worsens the stricture. When we perform gastric tube reconstructions, we always carefully perform end-to-side esophagogastric anastomoses, using a circular stapler to avoid bending the anastomosis, but regardless of this, strictures occasionally occur.

For anastomotic strictures, bougie dilations or endoscopic balloon dilation techniques are often performed, but in some cases, frequent dilation is required and the resultant expansion may still be insufficient. Additionally, new or 
recurrent cancer may occur in the esophageal remnant or at the anastomotic site. These complications indicate surgical treatment. However, in the case of cervical anastomoses, approaching the anastomosis is difficult because it is located on the dorsal side of the sternum, and advanced adhesions to the surrounding tissues are often encountered. For such cases, we have adopted a procedure to approach the anastomosis via the sternum; the procedure involves resection of the proximal left clavicle and manubrium.

\section{Technique}

First, an incision is made in the left half of the neck wound that resulted from the previous operation, followed by a midline incision (Fig. 1a). The platysma is peeled back and the sternocleidomastoid, sternothyroid, sternohyoid, and the left pectoralis major muscle are detached from the sternum and left clavicle (Fig. 1b). Release of the left sternoclavicular joint is accomplished by cutting through the center of the left clavicle with a wire saw, and removing the proximal left clavicle (Fig. 1c). Resection of the manubrium is then performed. Because the gastric tube is extensively adherent to the manubrium, the manubrium is carefully shaped, using Luer bone rongeur forceps, from the left proximal side of the manubrium to ensure an appropriate field of view (Fig. 1d), thereby maximizing patient safety. In the case of cancer, a neck dissection may be performed, if necessary. Subsequently, the esophagus and the gastric tube around the stricture are totally exposed and resected (Fig. 1e). Since the adhesion is not as severe around the proximal side of the remnant esophagus, the esophagus is encircled and lifted with cotton tape, prior to the anastomotic site being carefully detached. In this series of operations, care is taken to avoid damaging the left recurrent nerve. Occasionally, the left recurrent nerve is difficult to identify due to the severe adhesions; we attempt to stay as far away from the trachea as possible and promote the dissection along the esophagus and gastric tube.

When the excision range is small, re-anastomosis is possible but requires a free jejunal interposition. In the case of re-anastomosis, we perform an end-to-end manual anastomosis with the Albert-Lembert procedure. In the case of free jejunal interposition, we usually perform an end-to-side anastomosis for the proximal anastomosis and an end-to-end procedure for the distal one, using a circular stapler. The left cervical transverse artery, the left internal thoracic artery, the left internal or external jugular vein, or the left vertebral vein can be used for revascularization, depending on the position and length of the jejunal graft. Figure $1 \mathrm{f}$ shows a completed reconstruction. Finally, the surgical field is washed thoroughly with saline, vacuum drains are placed on both sides of the anastomosis, and the incised wound is sutured.

\section{Results}

This procedure was used for seven patients between April 2000 and March 2011; a summary of these cases is shown in Table 1. All cases were gastric tube reconstructions, via the retrosternal route. Three cases involved cancer of the esophageal remnant and four involved anastomotic strictures, with one of the anastomotic stricture cases being complicated by a gastric tube-bronchial fistula. The mean age of the patients was 69.9 years (range, 65-76 years) and all of the patients were men. The mean operative time was 506 min (range, 374-845 min), with an average blood loss of $297 \mathrm{ml}$ (range, 180-606 ml). Esophagogastric anastomoses were performed in two cases, and free jejunal graft transplantations were performed in five cases. For the arterial grafts, the left internal mammary artery was chosen in one patient and the left cervical transverse artery was chosen in four patients. For the venous grafts, the left external jugular vein was chosen in two patients, and the left internal jugular vein, the left vertebral vein, and the left internal thoracic vein were each chosen in one patient.

Surgical complications included jejunal graft necrosis in one patient (a reoperation was required); grade 2 dysphagia, by the Clavien-Dindo classification, occurred in two patients; and a deep, surgical site infection occurred in one patient. However, oral intake became possible in all cases, and none of the patients developed limited range of motion or other movement disorders of the neck and upper limbs as a result of the upper sternum and clavicle resections.

\section{Discussion}

In the case of a gastric tube reconstruction, leakage and scarring may occur at the site of the anastomosis, with an anastomotic stricture eventually forming. The bend in the anastomosis may worsen the stricture, leading to the patient's worsening nutritional status and an increased risk of aspiration. Certainly, these strictures decrease the postsurgical quality of life (QOL) for patients with esophageal cancer and also negatively impact the patient's prognosis. Therefore, when an anastomotic stricture develops, bougie dilation or endoscopic balloon dilations are commonly performed. One report has suggested that these dilation techniques have an initial success rate of $78-100 \%$, but approximately $50 \%$ of the patients require a repeat dilation. ${ }^{1}$ In some cases, the cancer may also recur or new cancer may develop in the esophageal remnant or anastomosis. In addition, some patients require frequent dilation or sufficient extension cannot be obtained even after repeated dilation. In patients with refractory benign esophageal strictures, electrocautery incisions, steroid injections, and esophageal stents ${ }^{2-4}$ have been reportedly used to dilate the 
Fig. 1 Schema of the operation. a An incision is made in the left half of the neck wound, created during the previous operation, along with a midline incision. b The sternocleidomastoid,

sternothyroid, sternohyoid, and the left pectoralis major muscle are detached from the sternum and left clavicle. $\mathbf{c}$ Release of the left sternoclavicular joint, by cutting the center of the left clavicle and removing the proximal left clavicle. $\mathbf{d}$ The manubrium is resected and shaped using Luer bone rongeur forceps, from the left proximal side. e Exposure and resection of the esophagus and gastric tube around the stricture. At first, the esophagus is encircled and lifted with a cotton tape.

The anastomotic site is carefully detached, being careful not to damage the left recurrent nerve. $\mathbf{f}$

Reconstruction is completed a

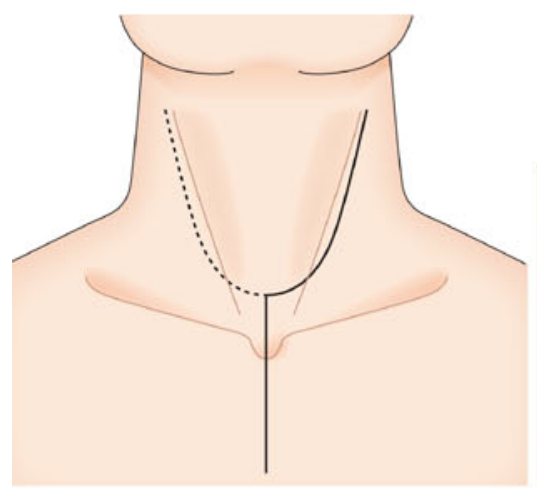

c

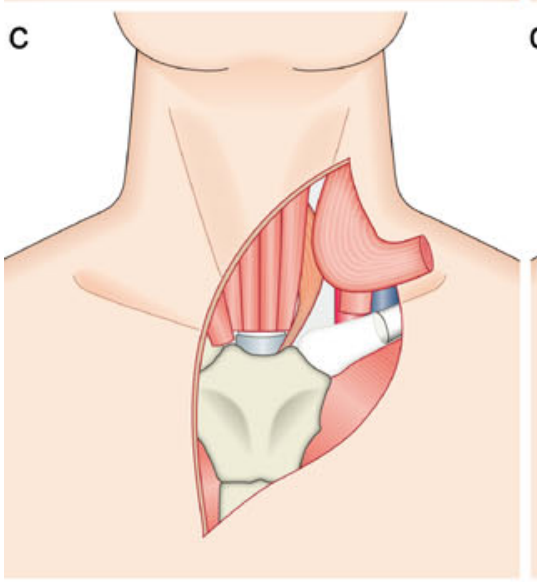

e

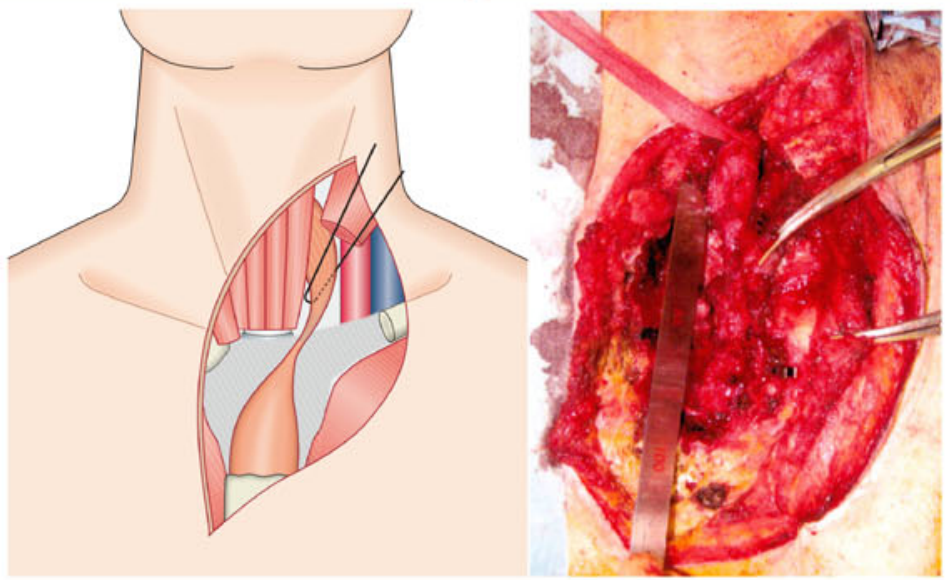

b

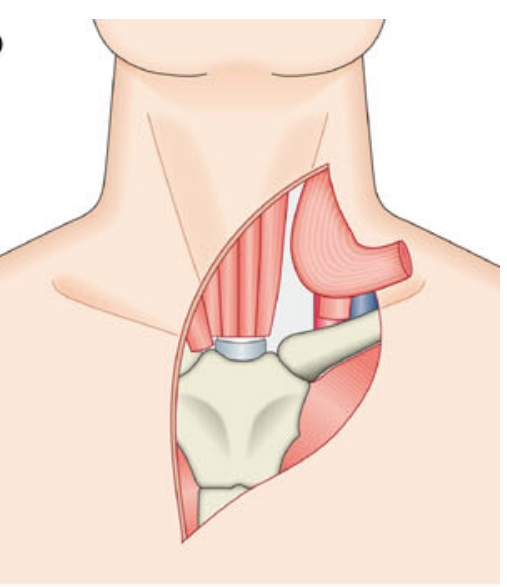

d

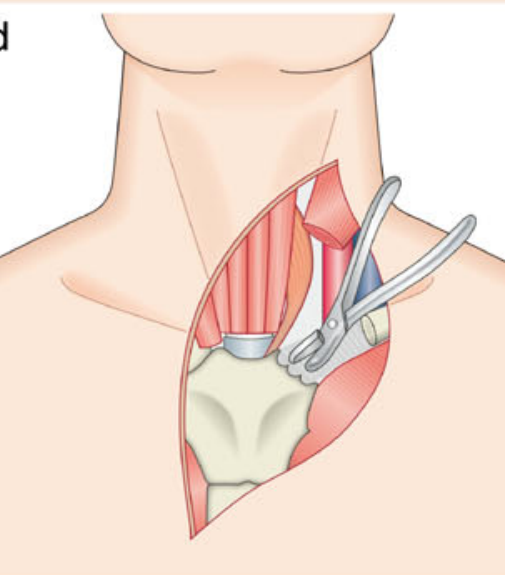

f

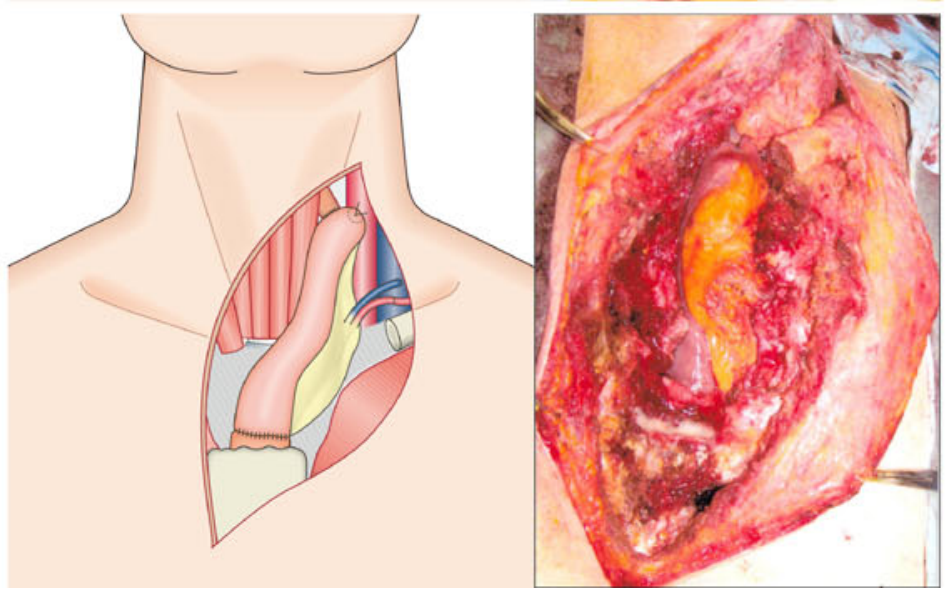




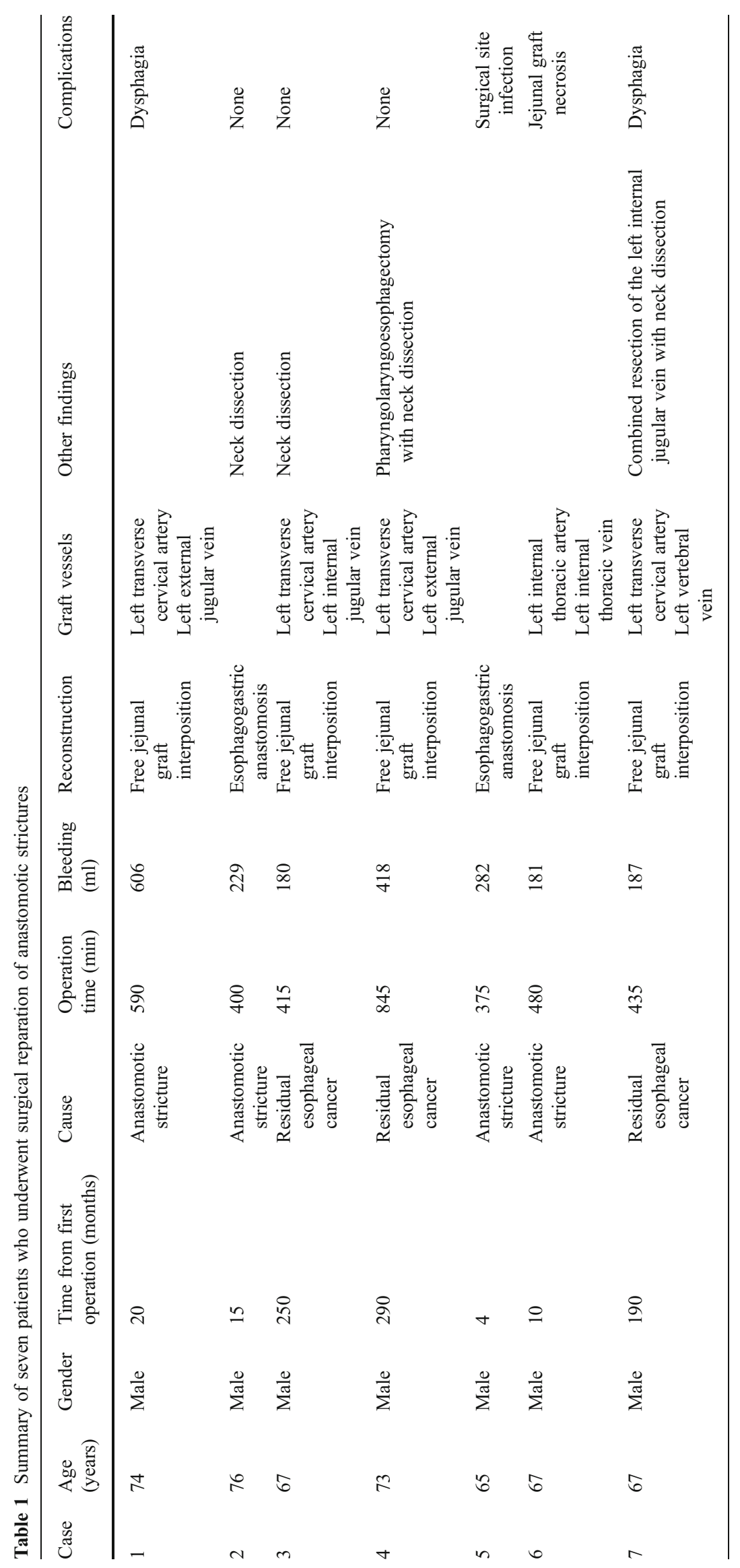


stricture. However, these techniques are limited when a long stricture is present or when it is bent.

In our division, anastomotic strictures are normally treated by endoscopic balloon dilation, but we have adopted the currently described surgical procedure for patients with severely degraded QOL, resulting from the complications described earlier. The operation takes approximately $500 \mathrm{~min}$, or longer, as it requires revascularization. Nonetheless, the intraoperative blood loss is only approximately $300 \mathrm{ml}$.

In the 1970s, Orringer has already reported that resection of the manubrium, adjacent clavicles and ribs provides access to the upper thoracic esophagus. ${ }^{5}$ One published report described a tracheal approach, with a manubrium resection. ${ }^{6}$ There is also a report of an approach to the anastomotic stricture involving a manubrium resection. ${ }^{7}$ Because resection of the manubrium and the left clavicle causes depression of the anterior chest wall, this procedure is cosmetically inferior to the manubrium resection approach. Resection of the manubrium and left clavicle allows the motion of the neck and the upper limbs to be preserved. In terms of the surgical procedure, if only manubrial dissection is performed, then a sufficient field of view cannot be obtained or the surgical field may be deepened. Hence, left clavicle resection is also performed to ensure a sufficient surgical field. If advanced adhesions are present, adhesiolysis between the manubrium and the anastomosis may become difficult or cause damage to other organs. At first, we removed the proximal left clavicle, which makes the dissection relatively easy, and preserves the field of view and the working space. The manubrium can then be excised, piece by piece, using Luer bone rongeur forceps from the left proximal side of the manubrium.

As a means of repairing a cervical esophagogastric anastomosis complication, we have adopted direct reanastomosis or free jejunal graft interposition. It is believed that stricturoplasty may be sufficient when the length of the stricture is short; however, the indications for stricturoplasty are known to overlap with the indications for other interventions, such as electrocautery incisions or esophageal stents. For these reasons, and to ensure a complete cure of the stenosis, we do not perform simple stricturoplasties because we want to ensure complete cure of the stricture. The described procedure may be an acceptable alternative for patients who have life-threatening consequences or a significant decrease in QOL and are resistant to conservative treatment.

Open Access This article is distributed under the terms of the Creative Commons Attribution License which permits any use, distribution, and reproduction in any medium, provided the original author(s) and the source are credited.

\section{References}

1. Stangl JR, Gould J, Pfau PR. Endoscopic treatment of luminal anastomotic strictures. Techniques in Gastrointestinal Endoscopy 2006; 8: 72-80.

2. Lee TH, Lee SH, Park JY, Lee CK, Chung IK, Kim HS, Park SH, Kim SJ, Hong SJ, Lee MS. Primary incisional therapy with a modified method for patients with benign anastomotic esophageal stricture. Gastrointestinal Endoscopy 2009; 69: 1029-1033.

3. Altintas E, Kacar S, Tunc B, Sezgin O, Parlak E, Altiparmak E, Saritas U, Sahin B. Intralesional steroid injection in benign esophageal strictures resistant to bougie dilation. Journal of Gastroenterology and Hepatology 2004;19:1388-1391.

4. Liu J, Hu Y, Cui C, Li Y, Lin X, Fu J. Removable, fully covered, selfexpandable metal stents for the treatment of refractory benign esophagogastric anastomotic strictures. Dysphagia 2012; 27: 260-264.

5. Orringer MB, Sloan H. Anterior mediastinal tracheostomy: indications, techniques, and clinical experience. The Journal of Thoracic and Cardiovascular Surgery 1979; 78: 850-859.

6. Chan YW, Yu Chow VL, Lun Liu LH, Ignace Wei W. Manubrial resection and anterior mediastinal tracheostomy: friend or foe? The Laryngoscope 2011; 121(7): 1441-1445.

7. Kinoshita Y, Udagawa H, Tsutsumi K, Ueno M, Mine S, Ehara K. Surgical repair of refractory strictures of esophagogastric anastomoses caused by leakage following esophagectomy. Diseases of the Esophagus 2009; 22: 427-433 